\title{
Carnets
}

Revue électronique d'études françaises de l'APEF

Première Série - 2 Numéro Spécial | 2010

Littératures nationales: suite ou fin. Résistances, mutations \& lignes de fuite

\section{Lignes de fuite entre mots et images: Henri Michaux et Nicolas Bouvier}

\section{Lénia Marques}

\section{(2) OpenEdition}

Journals

Édition électronique

URL : http://journals.openedition.org/carnets/5194

DOI : $10.4000 /$ carnets. 5194

ISSN : 1646-7698

Éditeur

APEF

Édition imprimée

Date de publication : 1 juin 2010

Pagination : 191-202

\section{Référence électronique}

Lénia Marques, «Lignes de fuite entre mots et images: Henri Michaux et Nicolas Bouvier », Carnets [En ligne], Première Série - 2 Numéro Spécial | 2010, mis en ligne le 16 juin 2018, consulté le 21 avril 2019. URL : http://journals.openedition.org/carnets/5194; DOI : 10.4000/carnets.5194

\section{(c) (i) (5)}

Carnets est mis à disposition selon les termes de la licence Creative Commons - Atribution - Pas d'utilisation commerciale 4.0 International. 


\title{
LIGNES DE FUITE ENTRE MOTS ET IMAGES \\ Henri Michaux et Nicolas Bouvier ${ }^{1}$
}

LÉNIA MARQUES

CEMRI - Universidade Aberta lenia.marques@ua.pt

\begin{abstract}
Résumé
L'œuvre écrite et visuelle de Henri Michaux (Bruxelles, 1899 - Paris, 1984) et de Nicolas Bouvier (Genève, 1929-1998) propose des lignes de résistance et de mutation. D'un caractère interculturel comme interartistique (la peinture pour Michaux; la photographie pour Bouvier), l'œuvre des deux voyageurs-écrivains-artistes raconte et suggère une panoplie de voyages, au cours desquels des lignes de fuite (Deleuze et Guattari, 1980) sont recherchées en permanence.
\end{abstract}

\begin{abstract}
The written and visual work by Henri Michaux (Brussels, 1899 - Paris, 1984) and by Nicolas Bouvier (Geneva, 1929-1998) suggests resistances and mutations. Characterized by their intercultural scope and the dialogue they propose between the arts (writing, painting and photography), these works give an account of a panoply of journeys, through which lines of flight (Deleuze and Gattari, 1980) are permanently nurtured.
\end{abstract}

Mots-clés: Henri Michaux, Nicolas Bouvier, Voyage, Arts, Lignes de fuite Keywords: Henri Michaux, Nicolas Bouvier, Travel, Arts, Lines of flight

\footnotetext{
${ }^{1}$ Cet article s'encadre dans un projet de post-doctorat financé par la Fundação para a Ciência e a Tecnologia. 
Gestes du défi et de la riposte

et de l'évasion hors des goulots d'étranglement

Henri Michaux

... mais des fautes, on en fera toujours de ce côté-ci de la vie.

Nicolas Bouvier

Parler de littératures nationales pour des auteurs tels que Henri Michaux (Bruxelles, 1899 - Paris, 1984) ou Nicolas Bouvier (Genève, 1929-1998) manquerait de pertinence, excepté dans ce que leur œuvre propose de résistance, de mutation et de lignes de fuite. ${ }^{2}$

Au cours de leurs nombreux voyages, autour du monde mais aussi dans leurs propres abîmes intérieurs, Michaux et Bouvier font preuve d'un regard attentif sur autrui et sur euxmêmes. L'appréhension du monde ne peut se faire que par le voyage, qui met en œuvre un processus de réflexion où la confrontation à autrui est exprimée par l'écriture et la peinture (Michaux) et l'écriture et la photographie (Bouvier).

Véritables points-charnières de la rencontre entre cultures et entre différentes expressions artistiques, les œuvres des deux artistes-écrivains-voyageurs suggèrent un parcours qui se développe sur trois axes: le voyage, le regard sur le monde et le rapport à l'altérité. Nous chercherons donc à comprendre la dynamique des lignes de fuite de ces œuvres au carrefour des trois axes énoncés.

\section{Configurations rhizomatiques de l'œuvre}

Rhizome: "tige souterraine pérennante, souvent allongée et horizontale."3

Dans Mille plateaux, Gilles Deleuze et Félix Guattari affirment que dans le rhizome "[i]l n'y a que des lignes" (Deleuze et Guattari, 1980: 15) qui s'entrecroisent, s'influencent et se délimitent mutuellement: "[e]lles se transforment, et peuvent même passer l'une dans l'autre. Rhizome" (Deleuze et Guattari, 1980: 248). Ainsi, outre les lignes que l'on pourrait considérer comme structurales (les lignes molaires et les lignes moléculaires), d'autres lignes, difficilement saisissables, surgissent: les lignes de fuite. ${ }^{4}$ En effet, les lignes de fuite sortent

\footnotetext{
${ }^{2}$ La conception de ligne de fuite, utilisée par Gilles Deleuze et Félix Guattari dans Mille Plateaux (1980), sera développée un peu plus loin.

${ }^{3}$ Définition proposée par le Dictionnaire de la langue française, Lexis, Larousse, daté de 1994.

4 Selon les auteurs, il y a donc trois types de ligne: "ligne de segmentarité dure ou molaire"; "ligne de segmentation souple ou moléculaire"; et "ligne de fuite" (Deleuze et Guattari, 1980: 239-242).
} 
du cadre de la structure, cherchent à rompre et contribuent à elles seules au phénomène de déterritorialisation:

D [déterritorialisation] est le mouvement par lequel "on" quitte le territoire. C'est l'opération de la ligne de fuite. Mais des cas très différents se présentent. La $D$ peut être recouverte par une reterritorialisation qui la compense, si bien que la ligne de fuite reste barrée: on dit en ce sens que la $D$ est négative. [...] Un autre cas se présente lorsque la $\mathrm{D}$ devient positive, c'est-à-dire s'affirme à travers les reterritorialisations qui ne jouent plus qu'un rôle secondaire, mais reste cependant relative, parce que la ligne de fuite qu'elle trace est segmentarisée, divisée en "procès" successifs [...]. (Deleuze et Guattari, 1980: 634) ${ }^{5}$

Ainsi, ces lignes qui se faufilent dans les constructions rhizomatiques, surtout celles qui se situent dans le cadre d'une déterritorialisation négative, "dégagent elles-mêmes un étrange désespoir, comme une odeur de mort et d'immolation, comme un état de guerre dont on sort rompu: c'est qu'elles ont elles-mêmes leurs propres dangers" (Deleuze et Guattari, 1980: 280). C'est dans ce sens que l'on peut lire Michaux quand il écrit, dans Émergences Résurgences, "mes peintures devaient se faire, avaient besoin de se faire, par le chemin du désordre, de la sauvagerie, de l'annihilation" (Michaux, 1972: 39).

Voilà donc le chemin choisi, ou plutôt le chemin qui s'avère nécessaire à la création, à l'expression du sujet, voire à sa vie. Les deux concepts de ligne de fuite et de déterritorialisation tracent la perspective selon laquelle nous regarderons brièvement l'œuvre de Henri Michaux et de Nicolas Bouvier, tout en nous y concentrant sur le voyage et les différentes manifestations artistiques.

\section{Les mouvements du voyage}

Le voyage, tel qu'il est conçu et exprimé par les deux voyageurs-écrivains, en mots comme en images, suggère des lignes de fuite, souvent fortement marquées. Au long de nombreuses années, Henri Michaux a construit une œuvre prolifique qui a assumé des formes diverses et qui a joui majoritairement de deux moyens d'expression: la peinture et l'écriture. Dans son œuvre, la fragmentation est un élément constant, dont la dynamique et l'expression gagnent corps de manières distinctes. ${ }^{6}$ Dans ce processus difficile et complexe, plusieurs effondrements sont mis en route: les notions de totalité, d'CEuvre et de genre sont

\footnotetext{
${ }^{5}$ La notion de "déterritorialisation" définie par les deux auteurs est fort complexe. Dans cette étude le terme sera utilisé dans le sens balisé dans cet extrait.

${ }^{6}$ II reste encore à faire une analyse détaillée sur le fragment dans l'univers de Michaux, un univers sans doute riche dans ses processus complexes et ses questionnements.
} 
minées; en outre, l'utopie de l'unité de l'être, basée sur une identité qui se construit en confrontation à celle d'autrui, est objet de questionnement et de déconstruction. Dans ce sens, l'œuvre de Michaux n'est finalement que le reflet de l'être intérieur:

Désir qui aboie dans le noir est la forme

multiforme de cet être

(Michaux, 1982)

Le désir se trouve ainsi derrière la mise en forme de l'être. Toutefois cette "forme" n'est pas unique, fermée, fixée; elle est elle-même multiple, plurielle, changeante: "L'être intérieur a tous les mouvements, il se lance à une vitesse de flèche, il rentre ensuite comme une taupe, il a d'infinies hibernations de marmotte. Quel être mouvementé!" (Michaux, 1998: 620621)

En effet, dans l'œuvre de Michaux, la rupture est constante et fait partie d'une philosophie subversive qui atteint, de prime abord, le texte et, dans une phase ultérieure, l'image. Dans ses écrits, le lecteur est frappé par la métafragmentarité, c'est-à-dire par les moments où le fragment réfléchit sur le fragment. ${ }^{7}$ Ainsi, cette métafragmentarité se présente comme un phénomène paradoxal, souvent violent à l'égard du texte lui-même (agissant ainsi en tant que ligne de fuite dans le texte). De fait, dans les écrits de Michaux, et notamment dans l'écriture de ses voyages, réels comme imaginaires, la métamorphose et la violence jouent dans un terrain fertile de mouvement continuel, où la fixité est refusée et où émergent constamment des lignes de fuite: "Mouvement, comme désobéissance, comme remaniement" (Michaux, 1972: 61).

Ce mouvement "désobéissant" surgit aussi dans ses œuvres picturales, le trait furtif, le signe qui se transforme, le trait insignifiant qui devient bête, qui devient homme... ${ }^{8}$ Tout est mouvement, tout est déplacement. Déplacement physique et déplacement psychologique. Le voyage se fait souvent à l'intérieur de lui-même; il s'agit d'un voyage de confrontation avec soi et avec l'autre; un voyage qui permet de regarder les autres qui peuplent le monde comme des traits, comme des visages fantomatiques...

Raymond Bellour, pensant spécifiquement à Un Barbare en Asie, ${ }^{9}$ fait référence à ce mouvement contraire à la situation de départ:

\footnotetext{
${ }^{7}$ La métafragmentarité est une notion que j'ai développée dans mon étude sur la poétique du fragment (Marques, 2011).

${ }^{8}$ Je fais référence ici à son poème Mouvements, publié pour la première fois, en 1951, avec soixante-quatre dessins et une postface (cf. Michaux, 1982).

${ }^{9}$ Texte de Michaux qui raconte ses impressions de voyage en plusieurs pays du continent asiatique. Ainsi, nous lisons "Un Barbare en Inde", "Un Barbare à Ceylan”, "Un Barbare en Chine”, "Un Barbare au Japon”, "Un Barbare chez les Malais" (Michaux, 1933).
} 
L'originalité de l'Orient de Michaux, qui préfigure par exemple le mouvement plus ténu que tracera, quarante ans plus tard, le Japon dans la vision de Barthes, est de fournir à la pensée un ébranlement attendu, qui permet avant tout à celui qui le vit de se décrocher de lui-même et de se retrouver ainsi, à jamais, autre. Le voyage en Orient devient le vecteur d'une irréductibilité fondamentale de la sensibilité à l'égard de ses valeurs d'origine: il s'agit moins de les enrichir ou de les dénier que de pouvoir continuellement les contrer, les délier, les défaire, les transformer. (Bellour dans Michaux, 1998: 1107)

Le voyage devient ainsi une nécessité pour le sujet, bien qu'une portion de douleur et de souffrance y soit inhérente. Processus difficile auquel le voyageur doit se soumettre, le voyage est en soi-même un véritable combat que Bouvier décrit dans Routes et déroutes en ces termes, tout en citant l'exemple de Michaux: ${ }^{10}$

Il est vrai que si vous voyagez en garde basse, pour prendre un terme de la boxe, vous prenez des coups que vous ne prenez pas si vous voyagez en garde haute, c'est-à-dire en ayant tout bétonné à l'avance. Mais dans ce cas, il ne se passe pas grand-chose. En garde basse, ça peut être dangereux. Michaux a très bien exprimé ça dans le magnifique petit texte qui s'intitule Ecuador. [...] [O]n perçoit très bien que c'est l'action conjuguée du déplacement dans l'espace et de l'éther qui a fait de lui un visionnaire." (Bouvier, 2004a: 1297-1298)

\section{La douleur du voyage}

Michaux est en effet ce visionnaire qu'après l'expérience d'Ecuador et d'Un Barbare en Asie a préféré, à un certain moment, d'écrire sur des voyages imaginaires, dans des pays lointains. ${ }^{11}$ Dans ses récits de voyages imaginaires, la douleur du voyage est ainsi transférée vers des personnages fictifs:

Mes pays imaginaires: pour moi des sortes d'États-tampons, afin de ne pas souffrir de la réalité.

En voyage, où presque tout me heurte, ce sont eux qui prennent les heurts, dont j'arrive alors, moi, à voir le comique, à m'amuser.

Mes "Emanglons", "Mages", "Hivinizikis" furent tous des personnages-tampons suscités par le voyage. (Plume disparut le jour même de mon retour de Turquie où il était né.) (Michaux, 1950: 161)

\footnotetext{
${ }^{10}$ II faut dire que, malgré le fait qu'ils ne se soient jamais rencontrés, Michaux était parmi les écrivains préférés de Bouvier, qui d'ailleurs le cite à nombreuses reprises dans ses notes, livres et entretiens.

${ }^{11}$ Cf. Les récits Voyage en Grande Garabagne, Au Pays de la Magie et Ici, Poddema (Michaux, 1967).
} 
II fait donc le déplacement fictif d'une douleur qui est effectivement présente, à l'image de ce jeu entre réel et fictif décrit par le poète Fernando Pessoa pour l'acte poétique. Dans son poème "Autopsicografia", Pessoa écrit:

O poeta é um fingidor.

Finge tão completamente

Que chega a fingir que é dor

A dor que deveras sente.

(Pessoa, 1998: 94) ${ }^{12}$

Si Michaux n'appelle pas ce passage de la douleur du réel au fictif "dissimulation", il faut dire pourtant que le processus dans son essence est le même: il crée des personnages pour mieux pouvoir endurer les douleurs, les obstacles, l'épuration, les chocs, les bouleversements et toutes les transformations imposées par le voyage.

\section{Plumer, élimer, devenir transparent...}

Dans l'œuvre de Nicolas Bouvier, tout ce processus d'évacuation de la douleur est moins éclatant, que ce soit par rapport à sa vie, aux voyages, à l'écriture ou à la photographie. Le voyage, si difficile soit-il, agit surtout comme un élément obligé du processus de "devenir" vide, purgé; un "devenir" qui participe de la disparition de l'être. Le superflu est perdu au profit de l'essentiel par un acte qui prétend permettre au monde de mieux pénétrer le sujet.

$\mathrm{Au}$ départ, le voyage surgit au jeune Bouvier comme une délivrance des étroits réseaux familiaux: "le voyage m'a sauvé. J'ai échappé aux fantômes du plumier natal" (Bouvier, 2004a: 1268). Acte de déterritorialisation, partir ne correspond pas cependant, selon Bouvier, à une révolte; "c'est une manière de respiration" (Bouvier, 1963).

Le voyage, exprimé par les mots et par les images fixées dans la pellicule, représente pour Bouvier la possibilité de créer ses propres lignes de fuite: il remet en cause le familier, sort du cadre habituel, cherche le renouvellement constant du quotidien et, par là, de l'Homme. ${ }^{13}$ Le voyage participe d'un cheminement vers la (re)découverte de l'essence humaine et est le moyen, par différentes manifestations artistiques, de perpétuer la quête d'une unité utopique, d'un absolu parfait et harmonieux. Toutefois, ce que l'on cherche n'est

\footnotetext{
12 "Feindre est le propre du poète/ Car il feint si complètement / Qu'il feint pour finir qu'est douleur/ La douleur qu'il ressent vraiment.", "Autopsychographie" (Pessoa, 2001: 575).

${ }_{13}$ À cet égard, Nicolas Bouvier partage la philosophie de Charles-Albert Cingria, sur lequel il a noté: "Pour l'observateur suraigu dressant l'oreille et voyant même la nuit avec une capacité d'émerveillement rarement prise en défaut le Monde est un spectacle magique, cosmique, inépuisable" (Bouvier, 2005: 53).
} 
pas toujours ce que l'on obtient: "Mais c'est le propre des longs voyages que d'en ramener tout autre chose que ce qu'on y allait chercher" (Bouvier, 2004b: 539).

Nicolas Bouvier va donc se livrer à la recherche d'une confrontation à autrui, au monde et surtout à un "soi-même" en dehors de son territoire habituel et familier. À chaque pas, tout est mouvement transformateur. Le voyage surgit ainsi comme un moteur - déclencheur du vide, "peut-être notre moteur le plus sûr" (Bouvier, 2001: 418) - qui travaille à la transformation de l'être. Une transformation directement liée à l'épurement et à l'humilité de l'être devant l'autre et devant le monde.

Dans ce sens, le Genevois refuse le voyage comme expérience d'accumulation d'épisodes exotiques qui rendraient le sujet un sapin de Noël, fort décoré:

On ne voyage pas pour se garnir d'exotisme et d'anecdotes comme un sapin de Noël, mais pour que la route vous plume, vous rince, vous essore, vous rende comme ces serviettes élimées par les lessives qu'on vous tend avec un éclat de savon dans les bordels. (Bouvier, 1996: 53-54)

Bouvier suit la maxime de Michaux, quand, dans Poteaux d'angle, ce dernier écrit: "Non, non, pas acquérir. Voyager pour t'appauvrir. Voilà ce dont tu as besoin" (Michaux, 1971: 10).

\section{Distances et unité cosmique}

Le parcours de Henri Michaux peut en effet être une trajectoire vers le lointain, un lointain qui est certes bien souvent ce lointain intérieur dont il a si aigûment écrit (Michaux, 1998). Les mots utilisés dans Déplacements Dégagements pour décrire le voyage qu'il fait dans ses dernières années en Belgique sont significatifs pour saisir l'étendue de son parcours et de son attitude face au voyage jusqu'à ses derniers jours: "Voyage qui n'en finissait pas, qui se tenait toujours à distance" (Michaux, 1985: 17). ${ }^{14}$ La vie est ce voyage qui semble infini et qui a le pouvoir (presque) magique de maintenir à distance l'individu de lui-même.

Dans ce court texte intitulé "Voyage qui tient à distance", dans Déplacements Dégagements (Michaux, 1985: 17-24), le temps présent du titre contraste avec le temps passé de l'extrait, où le sujet actif, en voyage, le voit comme un déplacement / distancement infini. Dans ce dernier titre, Déplacements Dégagements, est présent le désir de fuite constante au familier; une recherche constante de déterritorialisation.

\footnotetext{
${ }^{14}$ Marc Quaghebeur fait référence a cet écrit lors de son analyse de la présence de la Belgique chez Henri Michaux (Quaghebeur, 2001).
} 
C'est dans ce sens que la distance, la révolte, la réaction, le fait d'être "contre" ce qui l'entoure, sont des traits caractéristiques qui nous permettent d'approcher la poétique de Michaux d'après la notion de rhizome. Effectivement, les lignes de fuite sont marquées d'une manière plus frappante et plus déchirante que chez Bouvier. Son art est un art fragmentaire, de rupture proclamée. Les lignes de fuite y sont émergentes à tous niveaux.

Or cet éclatement constant, presque obsessif, contraste avec l'attitude apparemment plus tranquille de Nicolas Bouvier, dont l'acte d'écriture vise à capter, à saisir et, dans une certaine mesure, à exprimer tant que possible l'unité cosmique. La même description serait valable pour ses prises de vue. Les arts sont exactement le moyen privilégié pour capter cette essence difficile à saisir: "Pour moi, la photo est un art qui est beaucoup plus proche de la musique que de la peinture parce qu'il est lié à un instant qu'il faut saisir" (Bouvier, 1984). ${ }^{15}$

Une fois tracé ce panorama, il faut dire que dans l'unité imposante de son œuvre, la rupture, lorsqu'elle existe, a des configurations distinctes. Tel est le cas des ruptures et déchirements successifs dans Le Poisson-scorpion: rupture avec le lieu, avec ses gens, avec son aimée, avec le réel, enfin avec soi-même: "J'ai peur d'être passé en douce de l'autre côté du miroir, et que cette peur envahisse ce qui me reste de raison" (Bouvier, 1996: 167). Au niveau de l'écriture - du voyage littéraire - la division en chapitres, le passage lacunaire du temps et les ellipses sont d'autant plus des lignes de rupture qui cherchent des voies possibles en dehors des espaces familiers.

Ainsi, ces lignes de fuite, si elles sont présentées à l'intérieur même de son œuvre, vont parallèlement dans deux sens: vers l'extérieur (c'est-à-dire, par rapport aux changements qui y sont mis en œuvre: transformations de l'Homme, du monde, des voyageurs,...); et vers l'intérieur (transformations du sujet). On peut donc parler de mutation par l'œuvre plutôt que dans l'œuvre. Les lignes de fuite ne se trouvent pas ainsi au niveau de la production de l'œuvre proprement dite; où, lorsqu'elles existent, elles restent encore très voilées. Du moins en ce qui concerne l'écriture, l'œuvre de Bouvier est un ensemble que les lignes de fuite, dans ce qu'elles ont de plus dangereux, semblent ne pas pouvoir atteindre.

\section{De la fixité aux fluxes}

En ce qui concerne spécifiquement la photographie, pour mieux saisir la tension entre le familier et les lignes de fuite, tension qui crée les conditions pour la déterritorialisation,

\footnotetext{
${ }^{15}$ Bouvier a un lien très fort à la musique (cf. Guyader et al., 2008).
} 
nous aurons recours aux termes de studium et de punctum utilisés par Roland Barthes dans sa Chambre claire (Barthes, 1995). ${ }^{16}$

Si l'on essaie de distinguer ces deux notions à longs traits, l'on pourra dire que le studium correspond aux données perçues de façon à peu près similaire par différentes personnes, qui partagent une culture et une mentalité. Les regards ne se différencient pas: l'un regarde le même que l'autre; l'un perçoit le même que l'autre. Donc, "[l]e studium, c'est le champ très vaste du désir nonchalant, de l'intérêt divers, du goût inconséquent" (Barthes, 1995: 1126-1127).

D'autre part, le punctum dépend de la sensibilité de tout un chacun et est par conséquent subjectif. II s'agit de cet élément, ce détail de la photographie, qui nous touche, qui nous appelle. Cet élément perturbateur vient ainsi briser la lecture familière et inconséquente du studium:

Le second élément vient casser (ou scander) le studium. Cette fois, ce n'est pas moi qui vais le chercher (comme j'investis de ma conscience souveraine le champ du studium), c'est lui qui part de la scène, comme une flèche, et vient me percer. [...] Ce second élément qui vient déranger le studium, je l'appellerai donc punctum; car punctum, c'est aussi: piqûre, petit trou, petite tache, petite coupure - et aussi coup de dés. (Barthes, 1995: 1126)

Les photographies de Nicolas Bouvier sont marquantes par leur beauté; une beauté poétique qui advient du pouvoir de ce second élément. En effet, le punctum des photographies de Bouvier est une ligne de fuite vers l'extérieur; elle sort du cadre de la photographie et a des effets en dehors d'elle-même.

Par ailleurs, la fixité caractéristique de l'art photographique peut devenir tout autre: elle devient mobile, parce que les forces et les tensions sortent du papier, se libèrent (pour utiliser un mot cher à Michaux) du joug des mots, du moins dans une certaine mesure, et viennent frapper l'observateur. La fixité devient mouvement. C'est dans "cette opération à caractère magique" (Bouvier, 2004a: 1309) que tout se passe. La photographie, si elle est fixe, se propose aussi d'appréhender et de recréer un mouvement qui lui est particulier. Le visage joue ici, pour Bouvier mais aussi pour Michaux, un rôle fort particulier. Bouvier affirme à cet égard: "Le photographe cherche, à force de patience et d'humilité, à faire apparaître le contour intérieur d'un visage" (Bouvier, 2004c: 702).

\footnotetext{
${ }^{16}$ Pour mesurer les différents aspects du processus de déterritorialisation par rapport à la photographie, l'étude de Jean-François Guennoc, "Chambres noires, chambres claires: la photographie chez Nicolas Bouvier: un usage inquiet du regard et du monde", peut se révéler très utile, puisque l'auteur y analyse le discours de Nicolas Bouvier sur la photographie (Guennoc, 2006: 49-62).
} 
En regardant les photos de Nicolas Bouvier, l'impression qui demeure est d'harmonie, de tranquillité, d'émotion vive, de sentiment de communion profonde avec l'autre et avec le monde. Où seraient alors les lignes de fuite? Tout simplement, elles sont là, dans le bouleversement intérieur qu'elles provoquent; dans l'acte qu'elles représentent de capter l'autre, et dans leur pouvoir d'établir un dialogue avec soi-même, c'est-à-dire avec cet autre qui est de l'autre côté de la photographie. C'est là aussi en effet que Bouvier et Michaux se placent, encore une fois, sous le même signe. Comme l'écrit Michaux: "Là où auparavant régnait fixité, il n’y avait plus que flux" (Michaux, 1972: 92).

\section{En guise de conclusion}

Le voyage, physique, imaginaire ou intérieur, est un moyen de déterritorialisation constante. Le sujet cherche à sortir de soi-même, des cadres familiers; il fuit la sédentarisation de toutes ses forces. Dans Saisir, Michaux s'écrie: "Et du mouvement, car je ne veux pas de l'immobile - ou alors du mobile dans l'immobile" (Michaux, 1979: [2]).

La fuite à la sédentarisation est, chez Bouvier, plus artistique que réelle, dans le sens où, pour écrire, il fallait en quelque sorte se sédentariser. Comme l'acte d'écriture lui prenait beaucoup de temps, il n'écrivait pas sur le vif, mais après des années d'intériorisation. Ainsi, l'un des prix à payer pour pouvoir écrire était celui de rester "immobile", chez lui, encore que déchiré par l'urgence de prendre le large. La fixation guette et est une menace permanente:

Un atelier ou une chambre qu'on meuble, déjà stable, c'est changer en sédentaire le semi-nomade qu'on est resté.

Autre menace de fixation: la peinture elle-même créant, bien connu des peintres, un état de besoin. Voilà qui mettrait fin à mes voyages soudains, à mes départs en coup de vent.

Gare à l'assujettissement!

Hésitation. Transition.

(Michaux, 1972: 16)

Les lignes de fuite sont malgré tout essentielles à la survie du voyageur, de l'artiste, de l'humanité. Elles sont les lignes du rhizome qui permettent la déterritorialisation, par résistance et par mutation, dans une cartographie artistique qui conjugue différents modes d'expression - une cartographie qui est aussi celle du voyage et de la vie, jusque dans les espaces du silence: 
Il est vrai aussi que j'ai peur, ou aversion, ou malaise, car il y a partout - et je ne dois pas l'oublier - en quelque pays où l'on pénètre une sorte de tension superficielle qu'il faut forcer. Désagréable! désagréable! Vous avancez et la résistance est là, contre vous, ne cédant pas, comme le public d'un bar, où vient d'entrer un buveur d'eau ou un pasteur.

J'ai pourtant tellement besoin de voyager. Ah, si je pouvais vivre en télésiège, toujours avançant, toujours en de nouveaux pays, progressant sur des espaces de grand silence... (Michaux, 1962: 30-31) 


\section{Bibliographie}

BARTHES, Roland (1995). La Chambre claire. In CEuvres complètes, 1974-1980, t.III. Paris: Éditions du Seuil, pp. 1105-1200.

BOUVIER, Nicolas (1963). "L'Usage du monde", Émission télévisée "À livre ouvert", TSR (Télévision Suisse Romande) du 28/11/1963, avec le journaliste Maurice Huelin, URL: http://archives.tsr.ch/dossier-bouvier/personnalite-bouvier2, consulté le 15/03/2010.

BOUVIER, Nicolas (1984). "L'Image et ses leçons", Émission télévisée "Visiteurs du soir", TSR (Télévision Suisse Romande) du 17/02/1984, URL: http://archives.tsr.ch/dossierbouvier/personnalite-bouvier4, consulté le 15/03/2010.

BOUVIER, Nicolas (1996). Le Poisson-scorpion [1982]. Paris: Gallimard.

BOUvieR, Nicolas (2001). L'Usage du monde [1963]. Paris: Payot.

BOUVIER, Nicolas (2004a). Routes et déroutes [entretiens avec Irène Lichtenstein-Fall]. [1992]. In: Nicolas Bouvier. CEuvres. Paris: Gallimard, pp. 1249-1388.

BOUVIER, Nicolas (2004b). Chronique japonaise [1975]. In: Nicolas Bouvier. CEuvres. Paris: Gallimard, pp. 495-669.

Bouvier, Nicolas (2004c). Notes en vrac sur le visage. In: Nicolas Bouvier. CEuvres. Paris: Gallimard, pp. 701-706.

BOUVIER, Nicolas (2005). Charles-Albert Cingria en roue libre (textes réunis par Doris Jakubec). Genève: Zoé.

Deleuze, Gilles et GuATTARI, Félix (1980). Mille plateaux. Capitalisme et schizophrénie II. Paris: Les Éditions de Minuit.

GUENNOC, Jean-François (2006). "Chambres noires, chambres claires: la photographie chez Nicolas Bouvier: un usage inquiet du regard et du monde". In: Artful Deceptions: Verbal and Visual Trickery in French Culture. Oxford: P.I.E. Peter Lang, pp. 49-62.

GUYADER, Hervé et al. (2008). L'Oreille du voyageur. Nicolas Bouvier de Genève à Tokyo. Genève: Éditions Zoé.

MARQUeS, Lénia (2011). Pour une poétique du fragment: Charles-Albert Cingria et Paul Nougé. Bruxelles: P.I.E. Peter Lang (sous presse).

MichaUX, Henri (1933). Un Barbare en Asie. Paris: Gallimard.

MichaUX, Henri (1950). Passages. Paris: Gallimard.

MıCHAUX, Henri (1962). Vents et poussières: 1955-1962. Paris: Éditions Galerie Karl Flinker.

MichaUX, Henri (1967). Ailleurs. Voyage en Grande Garabagne, Au Pays de la Magie et Ici, Poddema. Paris: Gallimard.

MıCHAUX, Henri (1972). Émergences-Résurgences. Genève: Éditions d’Art Albert Skira.

MichaUX, Henri (1979). Saisir. Paris: Fata Morgana.

MiCHAUX, Henri (1982). Mouvements [1951]. Paris: Gallimard.

MıCHAUX, Henri (1985). Déplacements Dégagements. Paris: Gallimard.

MıCHAUX, Henri (1998). "Mouvements de l'être intérieur", Lointain intérieur. In: CEuvres complètes, t. I. Bibliothèque de la Pléiade. Paris: Gallimard, pp. 620-621.

PessoA, Fernando (1998). Ficções do Interlúdio (1914-1935). Lisboa: Assírio \& Alvim.

PESSOA, Fernando (2001). CEuvres poétiques [trad. Patrick Quillier]. Coll. Bibliothèque de la Pléiade. Paris: Gallimard.

QuAGHeBeuR, Marc (2001). "Cryptage" de la Belgique chez Michaux. Analele Universitatii Bucuresti, "Signé H.M." (coor. Laurent Rossion), Anul L-2001, pp. 9-24. 